\section{Epithelial expression of the hormone leptin by bovine skin}

Francesca Mercati, ${ }^{1}$ Cecilia Dall'Aglio, ${ }^{1}$ Ludovica Timperi, ${ }^{1}$ Paola Scocco, ${ }^{2}$ Elena De Felice, ${ }^{2}$ Margherita Maranesi ${ }^{1}$

${ }^{1}$ Department of Veterinary Medicine, University of Perugia

${ }^{2}$ School of Biosciences and Veterinary Medicine, University of Camerino, Italy

\section{Abstract \\ Leptin (Lep) stimulates keratinocytes to} proliferate, intervenes in the wound healing and participates to hair follicle morphogenesis and cycle. While it is secreted by skin structures including epidermis and hair follicles, intradermal adipose tissue also seems to have a role in Lep secretion and accordingly in the control of hair follicle growth in mice and humans. Lep was investigated in the skin of humans and laboratory animals but there are not data regarding bovine species. The aim of this work was to study the expression of Lep and its receptor (LepR) in the skin of bovine and, at the same time, to investigate the presence and extension of intradermal adipose tissue. A morphological evaluation of the skin was performed while the presence and localization of Lep and LepR were analyzed by RTPCR and immunohistochemistry. A high and thick dermis without adipocytes was observed. Hair follicles and sebaceous and sweat glands were located in the proximal part of the skin while a thick layer of connective tissue, lacking adipose cells, separated these structures by subcutis. RT-PCR evidenced the transcripts for both molecules. By immunohistochemistry, Lep and LepR were observed in the epidermis and hair follicles. Based on the absence of intradermal adipose tissue and the presence of both Lep and LepR in the epidermis and in the hair follicle epithelium, it can be posited that in bovine skin Lep participates to the control of epidermis growth and hair follicle cycle through a paracrine and autocrine mechanism.

\section{Introduction}

Leptin (Lep), the first adipokine to be discovered, ${ }^{1}$ is a 167 -amino-acid peptide encoded by the obese $(o b)$ gene. ${ }^{2}$ It is primarily secreted into the bloodstream by visceral and subcutaneous adipose tissue and acts as a cytokine, reducing food intake and increasing energy consumption. Leptin is also produced by several tissues including the placenta, the gastric mucosa, the mammary epithelium and skeletal muscle. ${ }^{3}$

Leptin acts by binding to LepR, a class I cytokine receptor ${ }^{4}$ that comprises six different isoforms (LepRa-f). These isoforms share an identical transmembrane domain and extracellular ligand binding domain but differ in their intracellular segment. ${ }^{5}$ The receptor is expressed in many peripheral tissues, indicating that it has diverse biological functions for Lep. In fact, Lep participates in a wide variety of physiological and physio-pathological processes mainly evidenced in humans and rodents but also studied in farm animals, including hematopoiesis, angiogenesis, reproduction, growth, insulin sensitivity, immunity and inflammation through endocrine, paracrine and autocrine mechanisms of action, and therefore it can be described as a cytokine-like hormone with pleiotropic actions. , $^{2,3}$ Accordingly, Lep is also considered to be an important factor involved in skin biology. ${ }^{6}$ It stimulates keratinocyte proliferation during skin repair ${ }^{7}$ and takes part in the regulation of wound healing processes. ${ }^{8}$ Leptin and its receptor are induced in skin after irradiation and in epithelial tumors following radiotherapy. ${ }^{9}$ Finally, Lep seems to be involved in the control of hair follicle (HF) morphogenesis and its cycles of growth, regression, and rest. ${ }^{6}$ In the skin, Lep is mainly produced by the epidermis and HF keratinocytes. ${ }^{6,10}$

In addition, some authors showed the primary action of intradermal adipose tissue (IAT) in mouse and humans as a key source of signaling molecules and adipokines, including Lep, that intervene in regulating HF cycle. ${ }^{11,12}$ Following studies on humans and laboratory animals, investigation on this molecule has also been performed in domestic animals, ${ }^{13,14}$ although research on the skin is still limited. ${ }^{15}$

In this work, the presence and localization of Lep and LepR were investigated in bovine skin in order to improve our knowledge about the biology of the integumentary system in this species.

\section{Materials and Methods}

\section{Skin sample collection}

Skin samples were taken from ten bovines intended for human food use, five of the Marchigiana breed and five of the Limousine one, regularly slaughtered at the abattoir in accordance with the European Union regulation on the protection of animals at the time of killing (Council Regulation (EC) No 1099/2009). Bovines were all 18-month-old males with a weight
Correspondence: Cecilia Dall'Aglio, University of Perugia, Department of Veterinary Medicine, Via San Costanzo 4, 06126 Perugia, Italy. Tel. +39.075 .5857635 . Fax. +39.075.5857631.

E-mail: cecilia.dallaglio@unipg.it

Key words: Hair follicle; epidermis; leptin receptor; immunohistochemistry; RT-PCR; bovine.

Contributions: FM, manuscript drafting; FM, LT, immunohistochemical studies; MM, RTPCR studies; FM, PS, morphometric evaluation; EDF, PS, manuscript revision collaboration; CDA, work supervision collaboration. All authors read and approved the final manuscript.

Conflict of interest: The authors declare that there is no conflict of interest that could be perceived as prejudicing the impartiality of the research reported.

Funding: This study was financed by the "Fondo Ricerca di Base 2014" of the University of Perugia, Italy.

Acknowledgments: The authors wish to thank Mrs. Gabriella Mancini for her excellent technical assistance. The authors also gratefully acknowledge the English language editing of the text carried out by Eleanor Fabri and Sheila Santolamazza.

Received for publication: 8 November 2018. Accepted for publication: 29 December 2018.

This work is licensed under a Creative Commons Attribution-NonCommercial 4.0 International License (CC BY-NC 4.0).

(C) Copyright F. Mercati et al., 2019

Licensee PAGEPress, Italy

European Journal of Histochemistry 2019; 63:2993 doi:10.4081/ejh.2019.2993

ranging between 600 and $650 \mathrm{~kg}$. The specimens were collected from the ventral cervical region after a visual examination to ensure that there were no cutaneous lesions.

\section{RNA extraction and reverse transcription}

Skin tissues promptly removed from each animal were thoroughly washed with saline solution, and then immediately frozen in liquid nitrogen and stored at $-80^{\circ} \mathrm{C}$ until the time to evaluate the transcript expression.

Total RNA was extracted from $50 \mathrm{mg}$ of each tissue sample. ${ }^{16}$ The frozen tissue sample was homogenized in $1 \mathrm{~mL}$ of TRIzol ${ }^{\circledR}$. Analyses of purity and total RNA quantification were performed using NanoDrop spectrophotometer

(ThermoFisher Scientific, Waltham, MA, USA). The 
integrity of each sample was assessed by electrophoresis of an aliquot of $3 \mu \mathrm{g}$ RNA in agarose gel using ethidium bromide staining. Total RNA was treated with DnaseI according to the manufacturer's instructions to prevent genomic DNA contamination. Five micrograms of total RNA were reverse-transcribed in $20 \mu \mathrm{L}$ of Superscript III Reverse transcriptase cDNA synthesis mix using random hexamers. Genomic DNA contamination was checked by performing the PCR procedure without reverse transcriptase. ${ }^{16}$

\section{RT-PCR amplification}

The PCR amplification was performed as described in previous methodological procedures ${ }^{17}$ with the use of $1 \mu \mathrm{L}$ of complementary DNA as template. The RT-PCR primers for Lep were: forward primer $5^{\prime}$ GGCTGTCCACAGGAGAAGAG -3' (position: 601-621) and reverse primer 5'AGTGAGAGGGAGCTGGAACA -3' (position: 814-834), Bos taurus Lep mRNA accession number: NM_173928, product size: 233 bp. With a detection purpose, primers design for LepR transcript evaluation was realized in a common region for the different isoforms: forward primer 5'CAATCACTTGCAGGAAGCAA -3' (position: 1362-1382) and reverse primer 5'TGACACAAGCTGGTGGAGAG -3' (position: 1568-1588), Bos taurus LepR mRNA accession number: NM_001012285.2, product size: 226 bp.

$\bar{C}$ ycling conditions consisted of an initial denaturizing cycle at $94^{\circ} \mathrm{C}$ for $75 \mathrm{~s}$ followed by 35 cycles for each target gene (Lep and LepR) at $94^{\circ} \mathrm{C}$ for $15 \mathrm{~s}, 60^{\circ} \mathrm{C}$ for $30 \mathrm{~s}, 72^{\circ} \mathrm{C}$ for $45 \mathrm{~s}$, and a final extension step at $72^{\circ} \mathrm{C}$ for $10 \mathrm{~min}$. The complete set of samples was processed in parallel in a single polymerase chain reaction (PCR), using aliquots of the same PCR master mix. The amplified PCR-generated products $(20 \mu \mathrm{L}$ of $25 \mu \mathrm{L}$ total reaction volume) were analyzed by electrophoresis on $2 \%$ agarose gel using ethidium bromide staining. All the reagents used for RT-PCR were acquired from Life Technologies Italia (Monza, MB, Italy). The amplified products, collected from agarose gel after electrophoresis, were purified with Nucleospin Extract II kit (Macherey-Nagel Inc., Bethlehem, PA, USA) and their identity confirmed by DNA sequencing using Sanger's method.

\section{Morphological evaluation}

For basic histology, skin samples were fixed in a $10 \%$ formaldehyde solution in phosphate buffered saline (PBS) $(0.1 \mathrm{M}, \mathrm{pH}$ 7.4) at room temperature for $24 \mathrm{~h}$, dehydrated and embedded in a paraffin wax. Five$\mu \mathrm{m}$-thick sections were mounted onto polyL-lysine coated glass slides and processed for staining with Haematoxylin and Eosin (H\&E). Additional samples of the dissected skin for each bovine were quickly immersed in cold isopentane and frozen in liquid nitrogen. Cryosections of $7 \mu \mathrm{m}$ were cut and stained with Oil Red O, a specific staining for identify neutral lipids, in order to determine the presence of adipocytes in the dermal compartment of bovine skin. ${ }^{18}$

To evaluate the thickness of the dermis and perform a morphometric analysis, the tissue sections were observed under a light microscope (Eclipse E800, Nikon Corporation, Tokyo, Japan) and photographed with a digital camera (Dxm 1200, Nikon Corporation). The images were used to measure both the height of the superficial layer of the dermis where hair follicles, sweat glands and sebaceous glands are placed, and total dermis thickness from the basal membrane of the epidermis up to the border of the subcutis. The images were processed using image analysis software (Lucia Measurement, Laboratory Imaging Ltd, Praga, Czech Republic).

\section{Immunohistochemistry}

For immunohistochemical analysis, skin samples were processed as described for basic histology. Immunohistochemistry was performed as follows. ${ }^{15}$ Sections were microwaved for $15 \mathrm{~min}$ in $10 \mathrm{mM}$ citric acid (pH 6.0) for antigen retrieval. Then, they were immersed for $10 \mathrm{~min}$ in a peroxidaseblocking solution $\left(3 \% \mathrm{H}_{2} \mathrm{O}_{2}\right)$ in order to inhibit endogenous peroxidase activity. Non-specific labeling was avoided by incubation with normal goat or horse serum diluted 1:10 for $30 \mathrm{~min}$ at room temperature. Subsequently, the sections were incubated overnight with the following primary antisera: rabbit anti-Lep (clone H-146) and goat anti-LepR antibody (clone M-18), both diluted 1:100 and purchased from Santa Cruz Biotechnology (Santa Cruz, CA, USA). On the second day, sections were incubated for $30 \mathrm{~min}$ with 1:200 biotin-conjugated secondary antibodies, respectively: goat anti-rabbit and horse anti-goat from Vector Laboratories (Burlingame, CA, USA) and Chemicon (Temecula, CA, USA). The reaction was detected with an avidin-biotin system and visualized with diaminobenzidine (DAB) as chromogen (both systems from Vector Laboratories). Nuclei were counterstained with Mayer's Haematoxylin. Between all incubation steps, except after normal serum, sections were washed with PBS. All steps, including primary antibody incubation, were performed at room temperature and the slides were incubated in a humid chamber.

Endothelial cells and nervous fibers were used as internal positive controls for Lep and LepR respectively. ${ }^{19,20}$ For negative control, the immunohistochemical procedure was performed without applying the primary antibody and also incubating sections with normal rabbit IgG (Novus Biological, Littleton, CO, USA). All sections were observed under a photomicroscope (Nikon Eclipse E800, Nikon Corp.; Tokyo, Japan) connected to a digital camera (Nikon Dxm 1200 digital camera) and images were examined by using an image analysis system (Lucia, Laboratory Imaging Ltd., Prague, Czech Republic).

\section{Results}

\section{RT-PCR amplification}

Messenger RNAs for Lep and LepR were expressed in the bovine skin samples examined (Figure 1). The RT-PCR, carried out with the specific primers, detected the presence of the expected bp products: 233 bp for Lep and 226 for LepR, respectively.

\section{Morphological evaluation}

Bovine skin was very thick, due to high extension of the dermis. The more superficial layer of dermis contained HFs, sebaceous glands and sweat glands immersed in connective tissue comprised of fine bundles of collagen fibers with numerous cells. This layer was much thinner than the underlying dermis composed of dense irregular connective tissue with larger and thicker bundles of collagen fibers and fewer scattered connective cells. The underlying layer was 3.98 and 3.39 times thicker than the upper

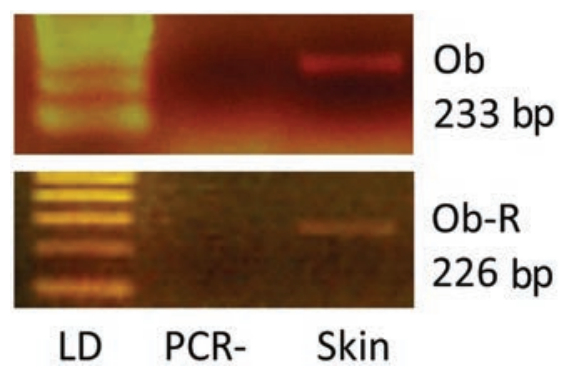

Figure 1. Representative photographs of typical $2 \%$ agarose ethidium bromide stained gels. The presence of the expected bp products yielded after RT-PCR using primers for target Lep (233 bp) and LepR (226 bp) is shown. Lane LD is the kilobase DNA marker, lane PCR- represents a negative control of non-reverse-transcribed RNA submitted to PCR amplification, while the other lane (Skin) identifies one of the bovine skin samples examined. 
one in Marchigiana and Limousine breed respectively (Figure 2). The bovine dermis morphometric evaluations are summarized in Table 1

Oil Red O staining for histological visualization of fat cells and neutral triglycerides showed the absence of adipocytes in the whole dermal compartment, both as single cells and as aggregates. Close examination of the perifollicular region, particularly near the bulb, did not reveal the presence of adipose cells. Oil Red $\mathrm{O}$ staining gave a positive outcome in the excretory ducts of sebaceous glands and a less intense staining in the cells of the glands, where it evidenced the waxy substance secreted by the glands (Figure 3).

\section{Immunohistochemistry}

As determined by immunohistochemistry, the expression of Lep and its receptor was evidenced in some structures of bovine skin including epidermis, HFs, and sweat glands.

Leptin was abundantly expressed by the epidermis. While all layers of cells were involved, the suprabasal layers expressed a stronger signal (Figure 4). Leptin was also expressed by the outer root sheath (ORS) of HFs (Figure 5). Staining mainly extended into the regions of the infundibulum and isthmus. The inner root sheath (IRS) was negative. Immunostaining persisted in all stages of HFs, though the positive cells were not uniformly distributed in the regressive HFs compared with the anagen ones (Figure 6). The LepR showed a staining localization and pattern similar to that of Lep (Figure 7). In the epidermis, LepR was observed in the cells of the basal layer. However, there were few positive cells and they were characterized by weak staining. As regards HFs, LepR was clearly observed in the ORS cells and it mainly extended from the insertion of the sebaceous gland duct to suprabulbar region. The bulb region was negative. Finally, immunohistochemical signal persisted throughout the hair cycle. Other than epidermis and HFs, immunostaining for Lep and LepR was observed in the basal cells of the proximal region of the sweat glands (Figure 7). These cells likely belonged to the squamous epithelium that lines the sebaceous gland duct. In all structures described, staining

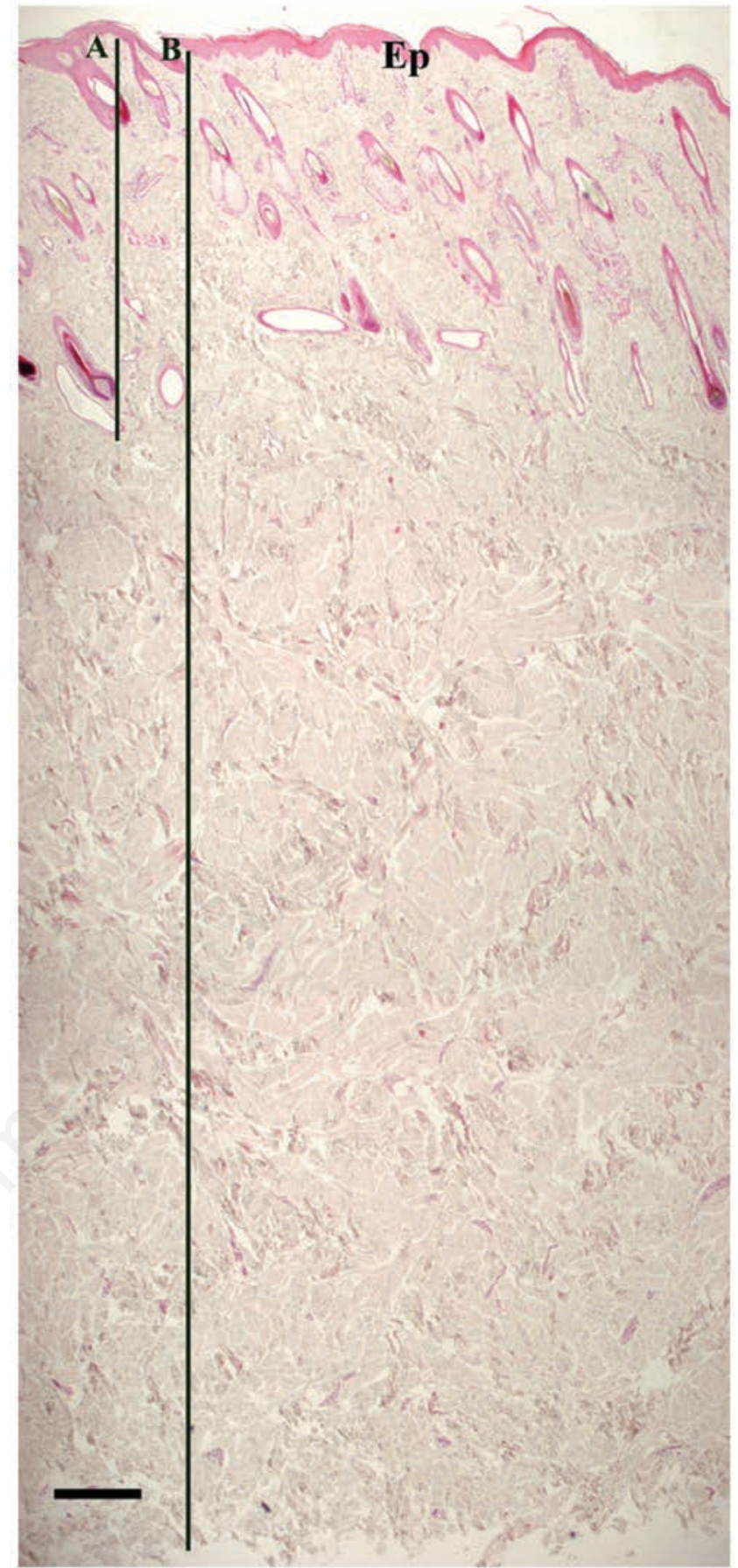

Figure 2. Low magnification of bovine skin. The dermis shows a great extension. The HFs and the glands can be observed in the more superficial layer, below the epidermis (Ep); this layer is pointed out by the bar " $A$ ". Bar " $B$ " points out the whole extension of the dermis, from the epidermis until the subcutis which has been removed. The photograph has been reconstructed. Haematoxylin \& Eosin. Scale bar: $400 \mu \mathrm{m}$.

Table 1. Morphometric evaluation of dermis. Dermis total height was measured from the basal membrane of the epidermis up to the border of the subcutis while the superficial layer was measured from the basal membrane of the epidermis to the proximal end of hair follicles and sweat glands.

\begin{tabular}{lcc} 
Bovine breed & Mean dermis total height \pm SD (jum) & Mean dermis superficial layer height \pm SD (jum) \\
Marchigiana & $5087.43 \pm 476.23$ & $1272.48 \pm 29.84$ \\
Limousine & $4467.29 \pm 334.96$ & $1325.29 \pm 142.05$ \\
\hline
\end{tabular}


was localized in the cytoplasm of the cells for both Lep and LepR. Vessel endothelial cells and nervous fibers in the dermis appeared positive for Lep and LepR; this reactivity was considered as internal positive controls (Figure 8). No staining was observed in the negative controls (Figure 8).

\section{Discussion}

Leptin is an interesting pleiotropic cytokine capable of exerting multiple actions. In order to fully understand the mode of action and the peripheral effects of this molecule, it is essential to investigate tissues and structures producing Lep and the peripheral distribution of its receptor. In this study, Lep and LepR m-RNAs were clearly detected in the skin of bovine by means of RT-PCR. Lep and LepR expression have been evidenced in a very high number of tissues in bovine species ${ }^{21}$ ${ }^{23}$ but never described in the skin.

In addition to RT-PCR, Lep and LepR were investigated by means of immunohistochemistry to observe their localization in the bovine skin structures. Strong and diffuse immunostaining for Lep was evidenced in the epidermis, as already described in humans. ${ }^{6}$ Instead, LepR was evidenced in the basal layer of the epidermis with the same localization described in the healthy skin of other species. $7,10,15,24$ The localization of the receptor in the proliferative compartment of the epidermis suggests a mitogenic action of Lep on the proliferative epidermal compartment in bovine species as well. ${ }^{7}$ Leptin may have an important role as a mitogen action on keratinocytes in both physiological epithelial turnover and following skin lesions such as irradiation. In a recent study, ${ }^{9}$ Lep mRNA was a possible target of miRNA during mouse skin irradiation and Lep itself was significantly modulated by irradiation in human epithelial cell lines. Lep and LepR detected in bovine skin may represent the existence of a potential defense system against different external insults.

Leptin and its receptor were clearly identified in the HFs throughout the follicular cycle. Both molecules were observed in the cells of the ORS, while the IRS was negative. In the bovine HF, as in the epidermis, the close proximity of the cells that express the molecule and its receptor suggests a paracrine and autocrine pattern of action by Lep. Studies have indicated that Lep, like other adipokines, acts through different mechanisms including endocrine, paracrine and autocrine action ${ }^{2,25}$ also in the skin. ${ }^{8,26}$ Lep and its receptor were observed in a large part of the bovine HF. Hoggard and others ${ }^{27}$ were the first to

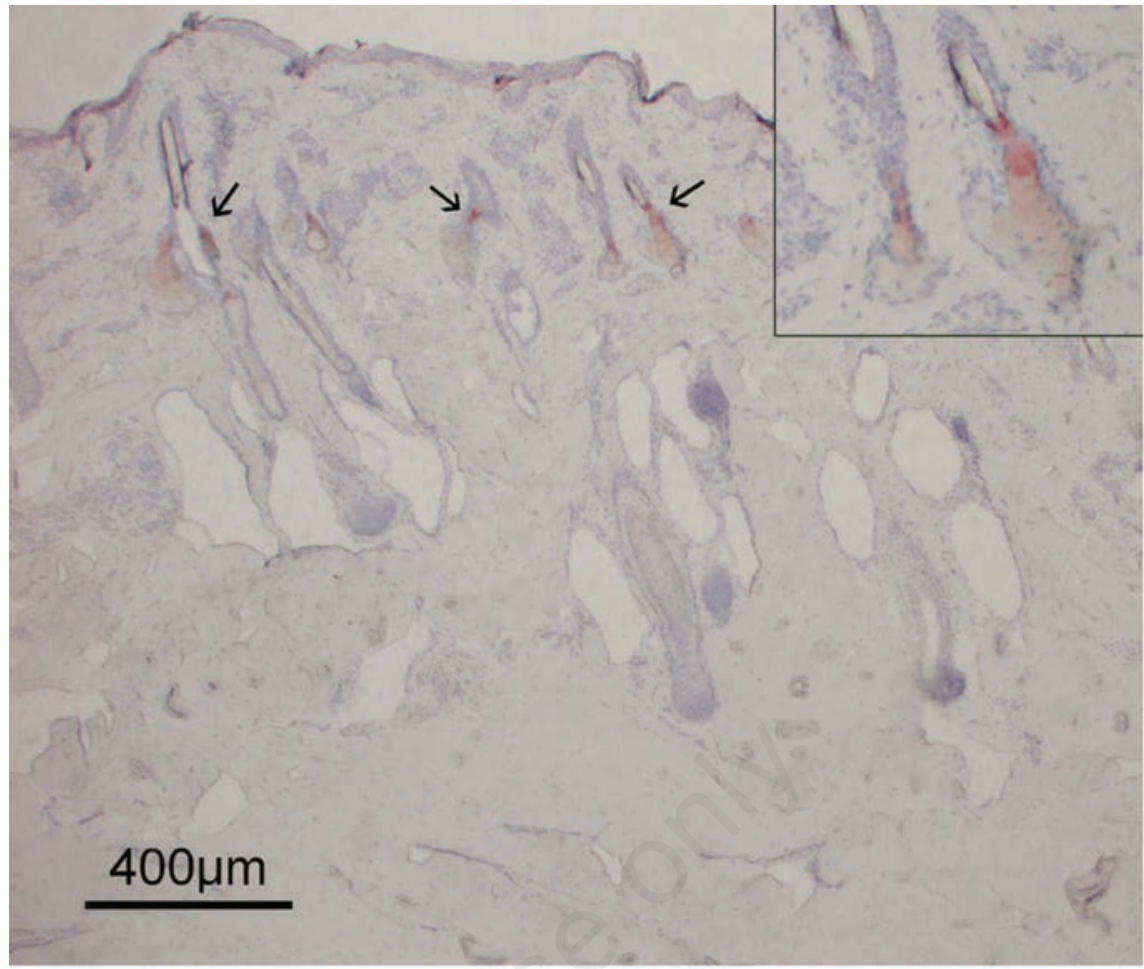

Figure 3. A section of bovine skin stained with Oil Red $O$ to visualize adipocytes. All the dermis appeared negative to this staining, attesting to the absence of adipose cells. Oil Red $O$ staining revealed the lipids in the inner part and the excretory duct of the sebaceous glands (arrows) localized in the upper part of the dermis. In the inset, a high-power magnification of two sebaceous glands positive to Oil Red O staining is shown. The gland ducts opening in the hair canals can be observed.

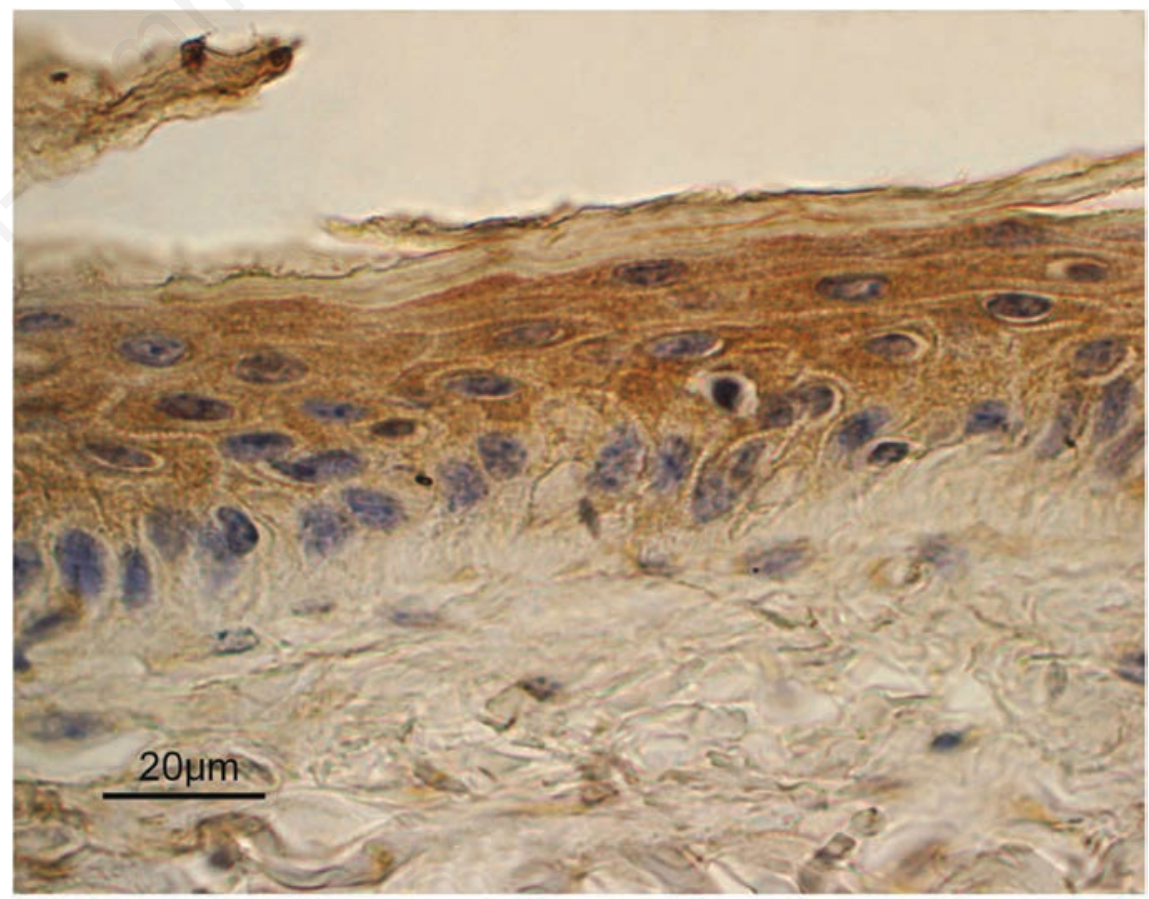

Figure 4. Lep expression in the epidermis. A diffuse staining was mainly observed in the suprabasal cell layers, compared to basal cells. ABC immunohistochemical staining; nuclei are counterstained with Haematoxylin. 
report a strong expression of these molecules in the HFs of the mouse throughout fetal developmental period, by means of in situ hybridization and immunohistochemical techniques. Successively, other authors have described Lep and LepR in the HFs with different results. $6,12,15,26,28$

It is known that six receptor isoforms exist deriving from an alternative splicing of the leptin receptor gene. ${ }^{5}$ Among these, LepRb is the only isoform with clearly demonstrated signaling capability through the activation of the Jak/STAT pathway, which allows signal transduction and transmission of leptin function; while, the other isoforms would be involved in leptin internalization, metabolism and turnover. ${ }^{5,29}$ However, the role of the short LepR isoforms is not quite known. ${ }^{5,30}$ Since the aim of the present study was to perform an early investigation on the leptin system in the bovine skin, both the primers and the LepR antibody were chosen based on their ability to evidence regions of the molecule common to all the receptor isoforms.

In addition to the skin, the adipose tissue is the main tissue secreting Lep, and circulating Lep is directly correlated with white adipose tissue mass. ${ }^{2}$ Leptin secretion by perifollicular adipocytes changes during follicular phases and a primary action of intradermal adipose tissue (IAT) on HF cycle was hypothesized. ${ }^{11,12}$ In this study, the morphological characteristics of the skin were evaluated in order to understand if IAT intervenes in follicular cycle through Lep secretion in bovine species, too. Morphological evaluation performed showed that no adipocytes were present in the dermal compartment, neither as a single cell nor as aggregates while, a very thick layer of dense irregular connective tissue, void of adipocytes, extended under HFs and the glands until subcutis. Dermis characteristics, included thickness, change according to breeds and body regions, ${ }^{31}$ however, bovine skin appears to be very different from mouse, where the HFs are abundantly immersed in the IAT. ${ }^{12,31}$ Based on the absence of IAT, it can be stated that the HF cycle in bovine is not controlled by the perifollicular adipocytes, as proposed for other species. Instead, the expression of Lep and its receptor in the HF epithelium allows us to suppose that HF acts as an endocrine organ which contemporaneously secretes the Lep hormone and undergoes to its action. In any case, we cannot exclude that the Lep secreted by visceral and subcutaneous adipose tissue can reaches the skin through the systemic circulation and to exerts an endocrine action on HFs.

In conclusion, the identification of the
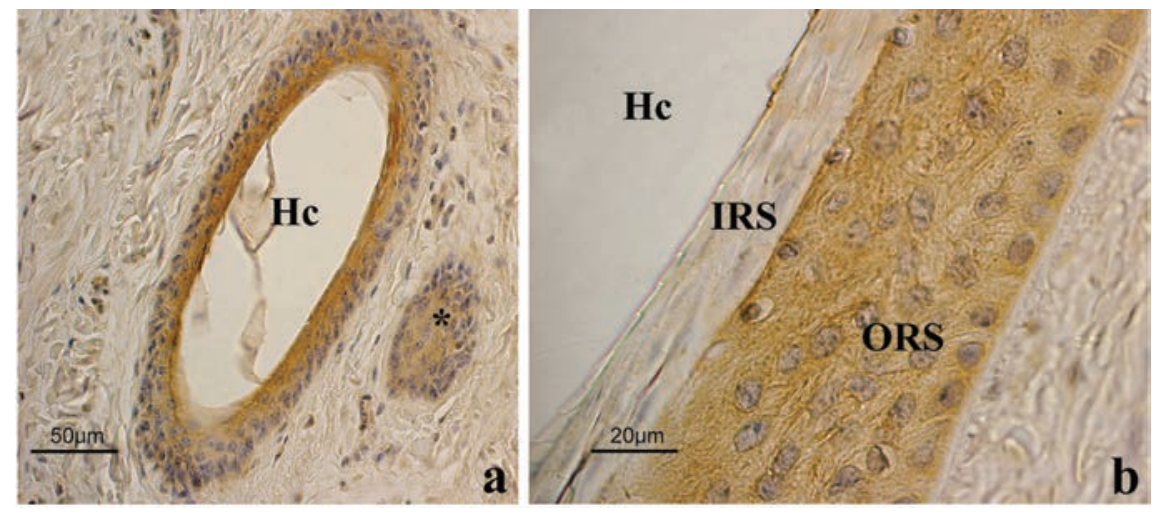

Figure 5. Lep immunohistochemistry in anagen HFs. a) Low magnification of an oblique section done at the level of the isthmus. Immunostaining involves the ORS cells. The positive structure $\left(^{*}\right)$ on the right is the wall of another HF. b) High power magnification of the isthmic region. Cytoplasmic staining of the ORS is clearly shown while the IRS appeared negative; $A B C$ immunohistochemical staining; nuclei are counterstained with Haematoxylin. ORS, outer root sheath; IRS, inner root sheath; Hc, hair canal.
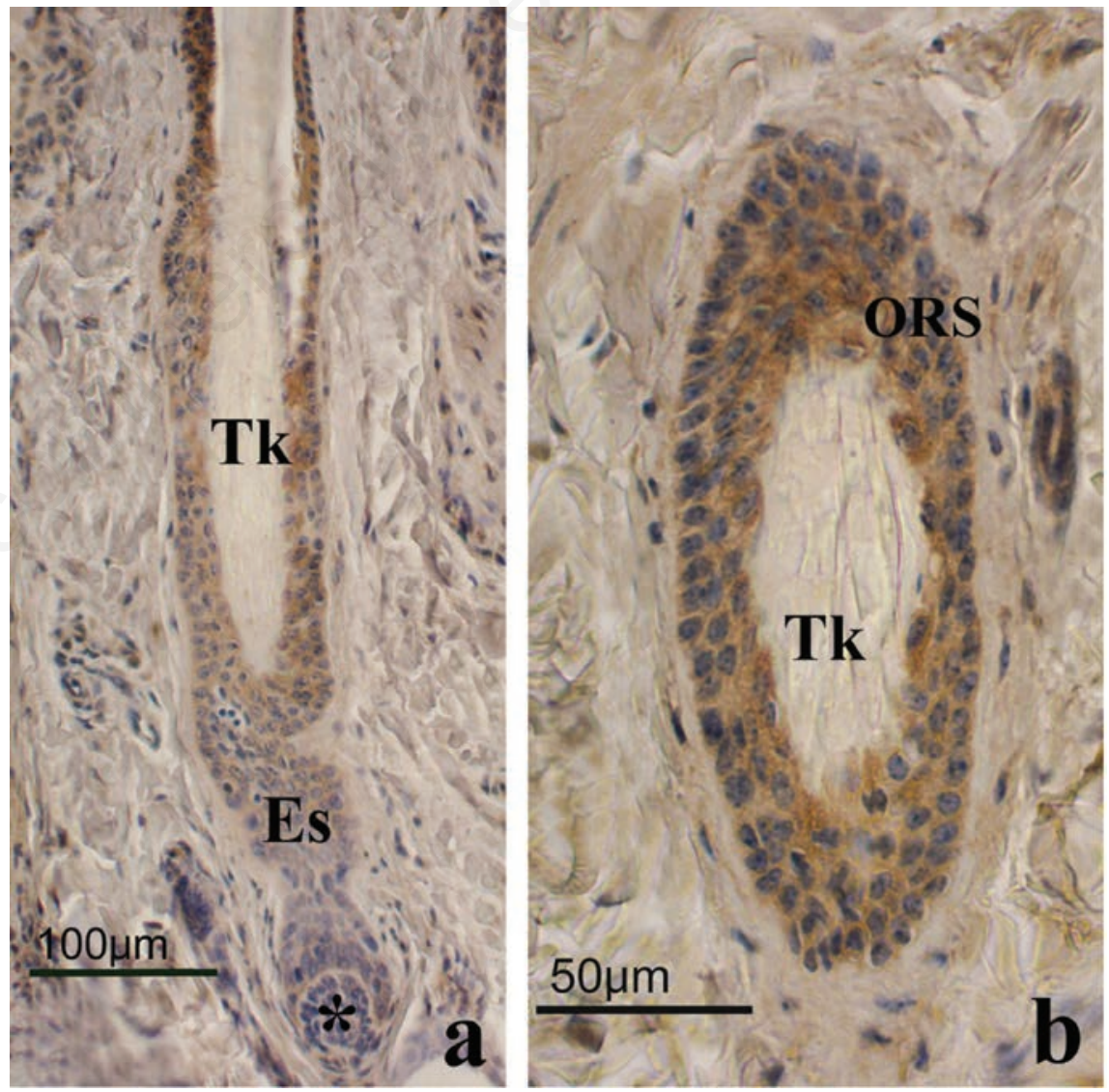

Figure 6. Lep immunohistochemistry in regressive phase HFs. a) Low magnification of a longitudinal section. Immunostaining involves the outer root sheath (ORS) cells, even if positive cells were unevenly distributed along follicular wall; the dermal papilla $\left(^{*}\right)$ is rounded and connected to the proximal part of HF by the epithelial strand (Es); the club hair showed the typical trichilemmal keratinization (Tk). b) High power magnification of a regressive phase HF; ORS showed a positive staining to Lep while trichilemmal keratinization (Tk) was clearly evident in the hair; $A B C$ immunohistochemical staining; nuclei are counterstained with Haematoxylin. 
Lep system in bovine skin provides important information for properly understanding the biological mechanisms that regulate skin, as well as for comparing animal species. The expression of LepR in the skin of bovine proves that Lep acts on this peripheral organ. The identification of Lep in the epithelial tissues of the skin attests that Lep may act through a paracrine and autocrine mechanism, even if an endocrine mechanism cannot be excluded. The absence of adipocytes around HFs and, broadly, in all the dermis indicates that the IAT does not exist in bovine and accordingly cannot exert paracrine control on the HFs.
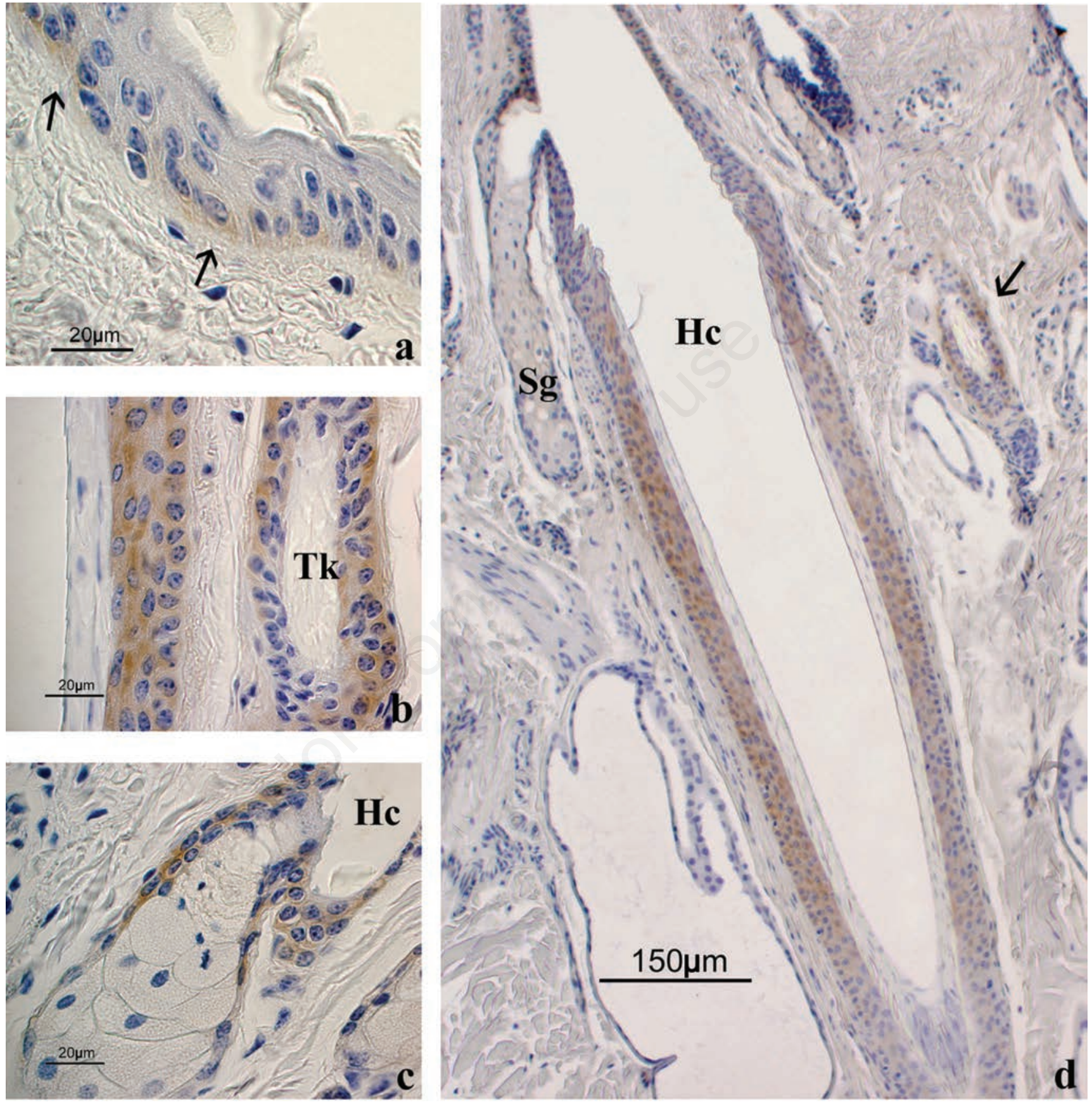

Figure 7. LepR immunohistochemistry. a) LepR expression in the epidermis; staining was observed in the basal layer (arrows) while the suprabasal cells were negative. b) High power magnification of an anagen HF (on the left) alongside a regressive HF (on the right); the outer root sheath cells of both follicles showed LepR immunostaining. c) LepR expression in a sebaceous gland; staining was observed in the squamous epithelium lining the sebaceous gland duct while secreting gland cells appeared negative. d) Low magnification of an anagen HF where LepR staining in the isthmus is shown; the arrow points to a regressive HF positive to LepR. Sg, sebaceous gland; Hc, hair canal; Tk, trichilemmal keratinization. ABC immunohistochemical staining; nuclei are counterstained with Haematoxylin. 

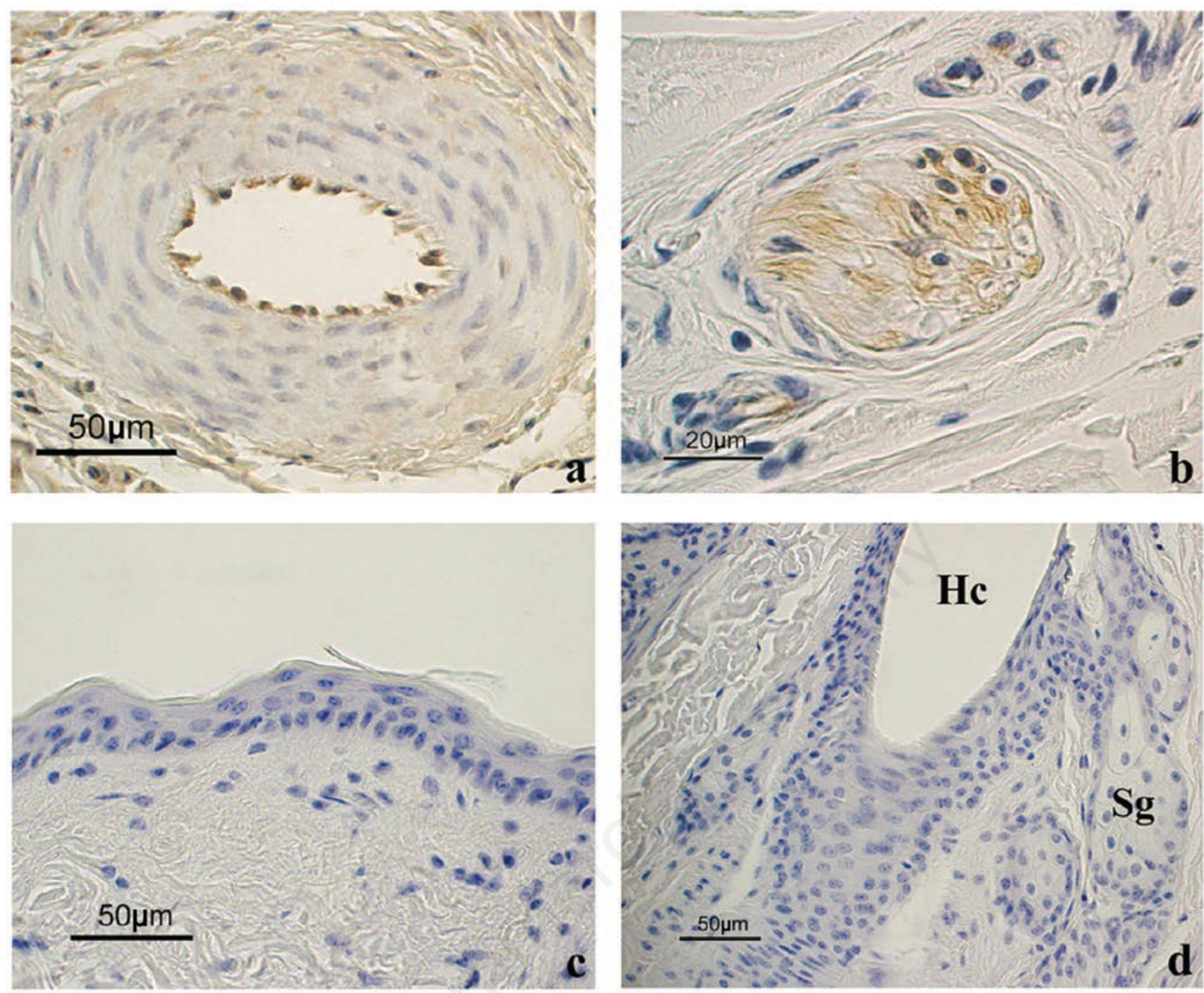

Figure 8. Positive and negative controls of the immunoreactions. a) Endothelial cells of a vessel in the dermis appeared positive to Lep. b) A nervous fiber in the dermis appeared positive for LepR. c) Negative control for Lep showing the epidermis and (d) for LepR showing a HF and sebaceous glands. The immunostaining is absent in all these structures. Hc, hair canal; Sg, sebaceous glands. All sections are counterstained with Haematoxylin.

\section{References}

1. Zhang Y, Proenca R, Maffei M, Barone M, Leopold L, Friedman JM. Positional cloning of the mouse obese gene and its human homologue. Nature 1994;372: 425-32.

2. Lago F, Dieguez C, Gómez-Reino J, Gualillo $\mathrm{O}$. The emerging role of adipokines as mediators of inflammation and immune responses. Cytokine Growth Factor Rev 2007;18:313-25.

3. Máčajová M, Lamosôvá D, Zeman M. Role of leptin in farm animals: a review. J Vet Med 2004;51:157-66.

4. Zabeau L, Lavens D, Peelman S, Eyckerman S, Vandekerckhove J, Tavernier J. The ins and outs of leptin receptor activation. FEBS Lett 2003; 546:45-50.

5. Dam J, Jockers R. Hunting for the functions of short leptin receptor isoforms. Mol Met 2013;2:327-8.

6. Poeggeler B, Schulz C, Pappolla MA, Bodò $\mathrm{E}$, Tiede $\mathrm{S}$, Lehnert $\mathrm{SH}$, et al. Leptin and the skin: a new frontier. Exp Dermatol 2010;19:12-8.

7. Frank S, Stallmeyer B, Kämpfer H, Kolb N, Pfeilschifter J. Leptin enhances wound re-epithelialization and constitutes a direct function of leptin in skin repair. J Clin Invest 2000;106:501-9.

8. Murad A, Nath AK, Cha ST, Demir E, Flores-Riveros J, Sierra-Honigmann MR. Leptin is an autocrine/paracrine regulator of wound healing. FASEB J 2003;17:1895-7.
9. Licursi V, Cestelli Guidi M, Del Vecchio G, Mannironi C, Presutti C, Amendola $\mathrm{R}$, et al. Leptin induction following irradiation is a conserved feature in mammalian epithelial cells and tissues. Int J Radiat Biol 2017;93:947-57.

10. Stallmeyer B, Kämpfer H, Podda M, Kaufmann R, Pfeilschifter J, Frank S. A novel keratinocyte mitogen: regulation of leptin and its functional receptor in skin repair. J Invest Dermatol 2001; 117:98-105.

11. Schmidt B, Horsley V. Unravelling hair follicle-adipocyte communication. Exp Dermatol 2012;21:827-30.

12. Yang CC, Sheu HM, Chung PL, Chang $\mathrm{CH}$, Tsai YS, Hughes MW, et al. Leptin of dermal adipose tissue is differentially expressed during the hair cycle and con- 
tributes to adipocyte-mediated growth inhibition of anagen-phase vibrissa hair. Exp Dermatol 2015 24:57-60.

13. Dall'Aglio C, Bazzucchi C, Mercati F, Ceccarelli P. Presence and distribution of leptin and its receptor in the minor salivary glands of the donkey. Acta Histochem 2015;117:305-8.

14. Dall'Aglio C, Mercati F, Pascucci L, Bazzucchi C, Ceccarelli P. Immunohistochemical evidence of leptin and its receptor in the carpal glands of domestic pigs and wild boar. Vet Dermatol 2015;26:46-8

15. Mercati F, Maranesi M, Dall'Aglio C, Scocco P, Pascucci L, Boiti C, et al. Leptin receptor is expressed by epidermis and skin appendages in dog. Acta Histochem 2014;116:1270-5.

16. Maranesi M, Zerani M, Lilli L, Dall'Aglio C, Brecchia G, Gobbetti A, et al. Expression of luteal estrogen receptor, interleukin-1, and apoptosisassociated genes after PGF2alpha administration in rabbits at different stages of pseudopregnancy. Domest Anim Endocrinol 2010;39:116-30.

17. Zerani M, Boiti C, Dall'Aglio C, Pascucci L, Maranesi M, Brecchia G, et al. Leptin receptor expression and in vitro leptin actions on prostaglandin release and nitric oxide synthase activity in the rabbit oviduct. J Endocrinol 2005;185:319-25.
18. Mazzi V. Manuale di tecniche istologiche e istochimiche. Piccin-Nuova Libraria, Milan; 1977.

19. Çerman AA, Bozkurt S, Sav A, Tulunay A, Elbaşi M, Ergun T. Serum leptin levels, skin leptin and leptin receptor expression in psoriasis. Br J Dermatol 2008;159:820-6.

20. Buyse M, Ovesjö ML, Goïot H, Guilmeau S, Péranzi G, Moizo L, et al. Expression and regulation of leptin receptor proteins in afferent and efferent neurons of the vagus nerve. Eur J Neurosci 2001;14:64-72.

21. Chelikani PK, Glimm DR, Kennelly JJ. Tissue distribution of leptin and leptin receptor mRNA in the bovine. J Dairy Sci 2003;86:2369-72.

22. Chilliard Y, Delavaud C, Bonnet M. Leptin expression in ruminants: nutritional and physiological regulations in relation with energy metabolism. Domest Anim Endocrinol 2005;29:3-22.

23. Kawachi H, Yang SH, Hamano A, Matsui T, Smith SB, Yano H. Molecular cloning and expression of bovine (Bos taurus) leptin receptor isoform mRNAs. Comp Biochem Physiol B Biochem Mol Biol 2007; 148:167-73.

24. Cao Y, Gao F, Li CZ, Xue YM. Expression of leptin and its long-form receptor in the marginal cutaneous tissues of diabetic foot ulcers. Acta Diabetol 2012;49:205-14.
25. Frühbeck G, Aguado M, Martinez JA. In vitro lipolytic effect of leptin on mouse adipocytes: evidence for a possible autocrine/paracrine role of leptin. Biochem Biophys Res Commun 1997;240:590-4.

26. Sumikawa Y, Inui S, Nakajima T, Itami $\mathrm{S}$. Hair cycle control by leptin as a new anagen inducer. Exp Dermatol 2014;23: 27-32.

27. Hoggard N, Hunter L, Duncan JS, Williams LM, Trayhurn P, Mercer JG. Leptin and leptin receptor mRNA and protein expression in the murine fetus and placenta. Proc Natl Acad Sci USA 1997;94:11073-8.

28. Iguchi M, Aiba S, Yoshino Y, Tagami H. Human follicular papilla cells carry out nonadipose tissue production of leptin. J Invest Dermatol 2001;117:1349-56.

29. Uotami S, Bjørbaek C, Tornøe J, Flier JS. Internalization and degradation of Leptin and ligand-induced receptor down-regulation. Diabetes 1999;48: 279-86.

30. Li Z, Ceccarini G, Eisenstein M, Tan K, Friedman JM. Phenotypic effects of an induced mutation of the ObRa isoform of the leptin receptor. Mol Metab 2013;2:364-75.

31. Driskell R, Jahoda CA, Chuong CM, Watt FM, Horsley V. Defining dermal adipose tissue. Exp Dermatol 2014;23: 629-31. 